\title{
Research on the MTBF evaluation method and the reliability improvement technologies of typical power adapter
}

\author{
Shen Zhong-hong, ${ }^{1,}$, Yang Lin ${ }^{1, b}$ and Jiang Chun- $\mathrm{u}^{1, \mathrm{c}}$ \\ ${ }^{1}$ The fifth research institute of MIIT, P.R.China \\ aszh@ceprei.biz, blynny@ceprei.biz, cjcx@ceprei.biz
}

Keywords: MTBF, Reliability improvement technologies, Manufacturing cost

Abstract. This paper researched about the MTBF evaluation method and the reliability improvement technologies of typical power adapter. Firstly, the basic structure of a typical power adapter was introduced; secondly, the reliability model was built, and the reliability parameter MTBF was evaluated; thirdly, some reliability improving technologies were researched to improve the reliability and reduce the manufacturing cost; lastly, the specific reliability data was compared between improvement and before. These reliability improvement technologies were effective and could be applied to design and manufacture high reliability electric products.

\section{Background}

With the development of science and technology, some power adapters with high performance and high security have emerged constantly, and had been used in almost all common electronic products. However, since the manufacturing companies are not at the same level in product design, the reliability indexes of different power adapters produced in different factories are very different especially the mean time between failures which named as MTBF, which ranged from nearly one hundred thousand hours to a few thousand hours. This situation made the further development of power adapter become difficult. Therefore, how to assess the reliability index MTBF of power adapter and how to improve the design faultiness have become the research focus of numerous power adapter manufacturers and research institutions relevant ${ }^{[1-4]}$. According to the statistics, the design defect is the first of all kinds of factors which affect the reliability of the electric products. Fig. 1 was the Fault data of electric products.

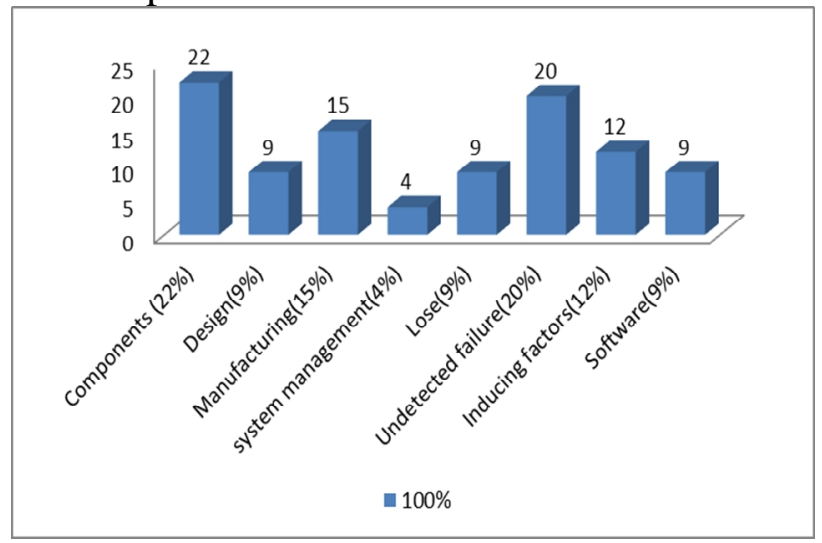

Fig. 1.(a) Fault data of electric products

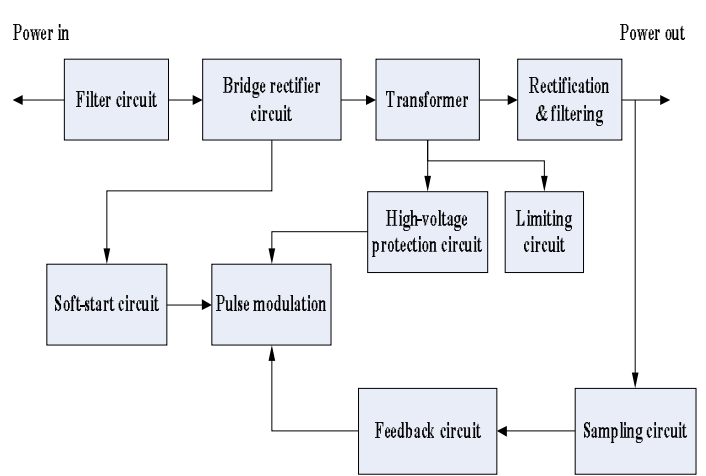

Fig. 1.(b) Fault data of electric products

\section{The basic structure of typical power adapter}

A typical power adapter mainly consisted of the transformer module, rectifier module, filter module, and regulator module, combined with protection module, feedback modules and other components. A typical circuit structure of a power adapter was shown in Figure 2. 


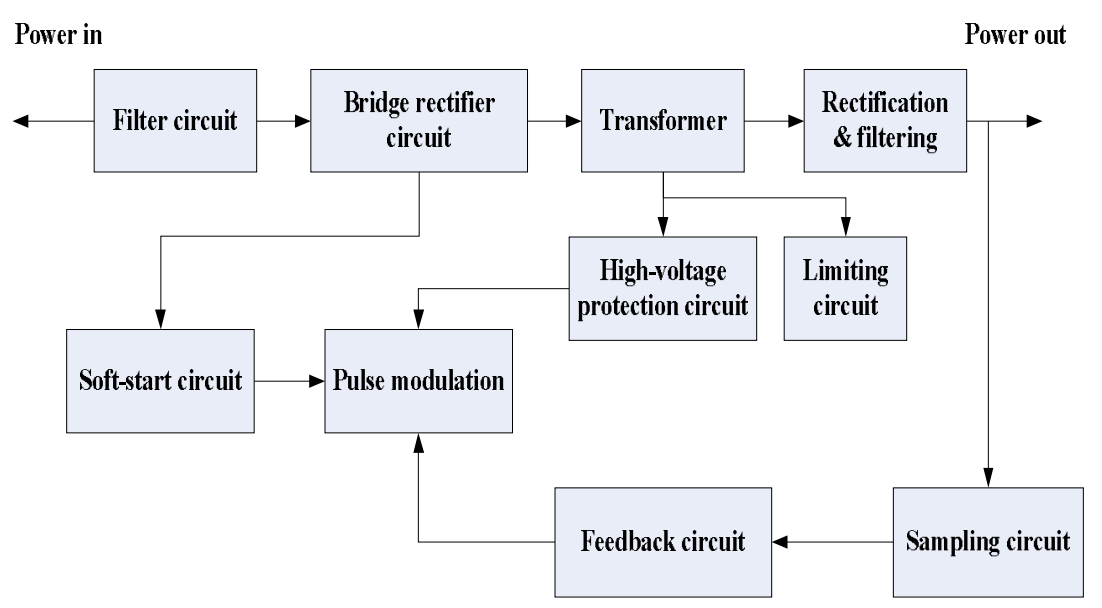

Fig. 2 A typical circuit structure of a power adapter

According to the typical circuit structure of a power adapter, a typical design schematics was shown as Figure 3.

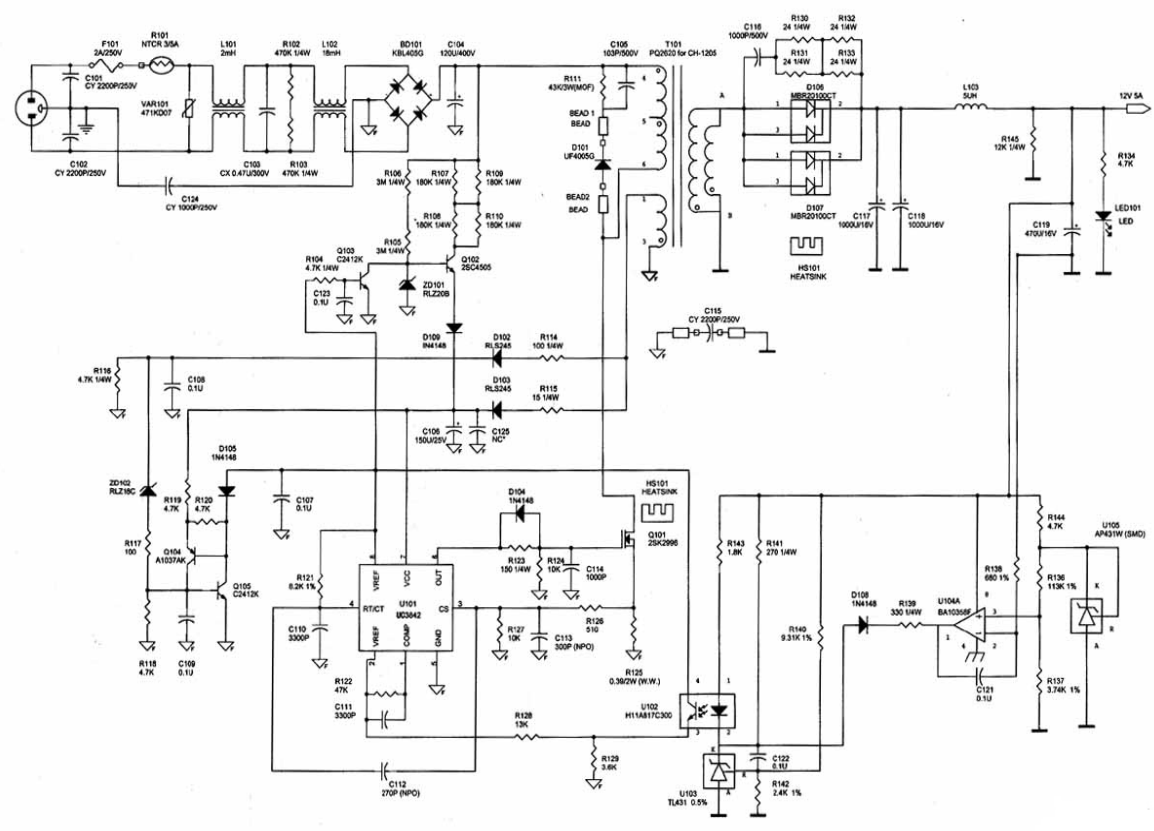

Fig.3 A typical design schematics of a power adapter

The main components of a power adapter contained resistors, capacitors, switching diodes, zener diodes, NPN transistors, integrated chips, thermistors, piezo-resistors, adjustable resistance, $\mathrm{X}$ capacitors, Y capacitors, photo-couplers, fuses, inductors, transformers, magnetic beads, PCB board and so on. The kind of electric components was nearly 20 and the amount was about 200.

The electrical parameters (voltage, current, power) and environmental parameters (temperature, humidity, etc.) of various components are not consistent when the power adapter working properly, the assessment methods about failure rate are inconsistent. Hence, how to assess the reliability index of the power adapter especially the MTBF index, was a complex task.

\section{The assessment method of MTBF of power adapter}

\section{The concept of MTBF}

The reliability was an important performance of a product. Reliability was the probability of a product which could complete a predetermined function in the given conditions and given time. The average life expectancy was the main gauge to measure the reliability of a product. For repairable products, the average life expectancy was an average time between failures of two adjacent, i.e. MTBF(Mean Time Between Failures). The MTBF was calculated by 


$$
M T B F=\int_{0}^{\infty} t f(t) d t
$$

Wherein, $f(t)$ was the instantaneous product failure rate.

For the products whose life distribution was exponential distribution, $\mathrm{MTBF}=1 / \lambda$, where, $\lambda$ was the total failure rate of the products. The life distribution of electronic products was exponential distribution usually. Based on this, we found that if we wanted to evaluate the MTBF of power adapter, the total failure rate $\lambda$ should be calculated first ${ }^{[5-8]}$.

\section{The reliability model of Power Adapter}

Expected failure rate of the power adapter was based on the establishment of its reliability models. Referring the functional principle of the device and system, divided the device into several parts which were relatively independent in circuit and whose internal units were tandem structure. And then made sure the logic and mathematical relationships between each divided parts, namely established reliability models of the products. The typical power adapter consisted of line filter, rectifier, transformer, filter, high-voltage protection circuit, pulse width modulation chip, limiting circuit, sampling circuit, the feedback circuit. The relationship between these various modules was series model.

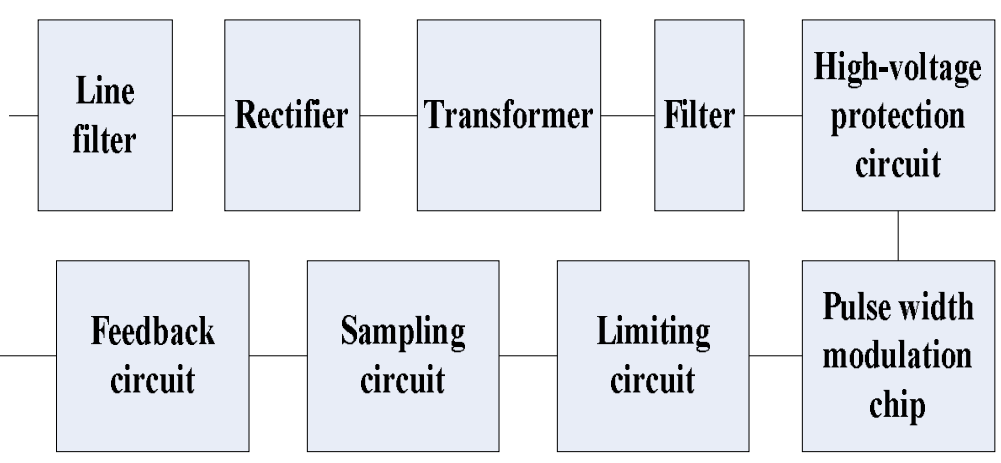

Fig.4 The reliability model of power adapter

\section{Stress analysis method}

According to the unit belonged, used ways, work temperature, electric stress and other environment parameters, connected with the failure model of electronic components and the reliability model of the product, the total failure rate of power adapter $\lambda$ could be obtained ${ }^{[9-10]}$.

$$
\lambda=\sum_{i=1}^{n} \lambda_{i}=\sum_{i=1}^{n} f_{i}\left(\lambda_{b}, \pi_{Q}, \pi_{T}, \pi_{V}, \pi_{C}, \pi_{E}, \pi_{L}, \pi_{A}\right)
$$

For the products whose reliability model was parallel structure, the $\lambda$ was:

$$
\lambda=\lambda^{\prime}\left(\frac{1}{1+\frac{1}{2}+\frac{1}{3}+\ldots+\frac{1}{n}}\right)
$$

\section{The improved technologies for MTBF}

\section{Increase the backup unit}

The failure rate was $2 / 3$ of the original failure rate when add a backup unit usually. If necessary, 1-2 units could be used as backup redundancy units.

The reliability of system was:

$$
R_{s}=1-F_{s}=1-\prod_{i=1}^{n} f_{i}=1-\prod_{i=1}^{n}\left(1-r_{i}\right)
$$

For the products whose life distribution was exponential distribution, MTBF was:

$$
M T B F=\sum_{i=1}^{n} \frac{1}{\lambda_{i}}-\sum_{1 \leq i<j \leq n} \frac{1}{\lambda_{i}+\lambda_{j}}+\ldots+(-1)^{n-1} \frac{1}{\lambda_{1}+\lambda_{2}+\ldots+\lambda_{n}}
$$


When $\mathrm{n}$ parts of the product were same, MTBF was:

$$
\text { MTBF }=\int_{0}^{\infty}\left\{1-\left(1-e^{-\lambda t}\right)^{n}\right\} d t=\frac{1}{\lambda}+\frac{1}{2 \lambda}+\ldots+\frac{1}{n \lambda}
$$

\section{Derating stress design}

Extra stress existed in some components. Thermal analysis showed that the ambient temperature of metal film resistors was $76{ }^{\circ} \mathrm{C}$, but the rated temperature was only $70{ }^{\circ} \mathrm{C}$, therefore, a higher rated temperature $\left(100{ }^{\circ} \mathrm{C}\right.$ above) needed to be considered for the metal film resistance.

Another example:

The original power load factor of one diode $\mathrm{S}$ was 0.4 , stress analysis showed that the ambient temperature was $80{ }^{\circ} \mathrm{C}$, the junction temperature was:

$$
T_{j} \approx 80^{\circ} \mathrm{C}+0.4 \times 150^{\circ} \mathrm{C}=140^{\circ} \mathrm{C}
$$

Its junction temperature exceeded the maximum allowable temperature $125{ }^{\circ} \mathrm{C}$ as Class II derating standard, so its load factor should be considered from 0.4 down to 0.25 or less.

System reliability optimization based on Lagrange allocation method

When MTBF and $\lambda$ could not reach the requirement value, or the manufacturing cost was too high when MTBF and $\lambda$ were fit, Lagrange allocation method could be used to realize the reliability allocation, and which made the manufacturing cost least.

Supposing there were $\mathrm{n}$ sub-system in the power adapter, the reliability of each sub-system was $\mathrm{R}_{\mathrm{i}}(\mathrm{i}=1,2, \ldots, \mathrm{n})$, the manufacturing cost was $\mathrm{x}_{\mathrm{i}}$, and the relationship between $\mathrm{R}_{\mathrm{i}}$ and $\mathrm{X}_{\mathrm{i}}$ was:

$R_{i}=1-e^{-\alpha_{i}\left(x_{i}-\beta_{i}\right)}$

The limit condition was the total reliability $R_{s}=\prod_{i=1}^{n} R_{i}$ was immovable. For solving this, Lagrange multipliers $\S$ was introduced to structure Lagrange function as follow:

$$
\begin{aligned}
& L(X, \xi)=\sum_{i=1}^{n} x_{i}-\xi\left(R_{s}-\prod_{i=1}^{n} R_{i}\right) \\
& x_{i}=\beta_{i}-\frac{\ln \left(1-R_{i}\right)}{\alpha_{i}} \\
& L(R, \xi)=\sum_{i=1}^{n}\left[\beta_{i}-\frac{\ln \left(1-R_{i}\right)}{\alpha_{i}}\right]-\xi\left(R_{s}-\prod_{i=1}^{n} R_{i}\right)
\end{aligned}
$$

Step the formula (12) into equation (11), then

$$
L(R, \xi)=\sum_{i=1}^{n}\left[\beta_{i}-\frac{\ln \left(1-R_{i}\right)}{\alpha_{i}}\right]-\xi\left(R_{s}-\prod_{i=1}^{n} R_{i}\right)
$$

$\left\{\begin{array}{l}\frac{\partial \text { King these equations: }}{R}=0 \\ \frac{\partial L}{\xi}=0\end{array}\right.$

Then the reliability value of each sub-system $R^{*}=\left[R_{1}^{*} R_{2}^{*} \ldots R_{n}^{*}\right]$ when the cost was least and the reliability was best.

\section{Test}

We chose one typical power adapter for testing. The total failure Rate of the power adapter before improvement was $50 \times 10^{-6} / \mathrm{h}$, MTBF was $20000 \mathrm{~h}$, manufacturing cost was about 135 Yuan. And The 
total failure Rate of the power adapter before improvement was $35.9 \times 10^{-6} / \mathrm{h}$, MTBF was $27855 \mathrm{~h}$, manufacturing costs was about 95 Yuan by using redundant backup, stress derating design, system reliability optimization and some other methods. It could be found that the MTBF of the power adapter had been greatly improved and the manufacturing cost was reduced in a certain extent after improving the reliability.

\section{Summary}

Using redundant backup and stress derating design approach can improve the reliability of electronic products. Applying optimization method for reliability system can reduce manufacturing costs. These methods can be applied to design and manufacture other electronic products.

\section{References}

[1] Y. Harada.T. Kurashiki.; N. Waris, M. Zako. Estimation of failure rate of consumer products based on components failure rate and its product safety assessment[C]. 2013 IEEE Symposium on Product Compliance Engineering (ISPCE): $1-5$.

[2] W J Xue, G G Jing L T Hong. Reliability improvement of electric power steering system based on ISO 26262[C]. 2013 International Conference on Quality, Reliability, Risk, Maintenance, and Safety Engineering (QR2MSE):125-129.

[3] L Xiao, R Yi, Z L Wang. Modeling method of SysML-based reliability block diagram[C]. Proceedings 2013 International Conference on Mechatronic Sciences, Electric Engineering and Computer (MEC):206 - 209.

[4] P Wang, X Yu, G Mai, etc.The interconnect reliability correlation with design and transportation stress[C]. 2013 14th International Conference on Electronic Packaging Technology (ICEPT),:595 - 599.

[5] T.E. Turner. Interconnect reliability assurance for circuits with billions of transistors[C]. 2013 20th IEEE International Symposium on the Physical and Failure Analysis of Integrated Circuits (IPFA):149 -151 .

[6] H B Zhang, L Liu. Reliability design of CNC system[C]. 2011 IEEE 18Th International Conference on Industrial Engineering and Engineering Management (IE\&EM), 2011(2):1140 - 1142.

[7] X Y Ren,Y Q Wu, X G Luo. Reliability design and analysis method under digital development environment[C]. Prognostics and System Health Management Conference (PHM-2014 Hunan): $441-445$.

[8] H Zhang. Talking about the reliability design of PLC control system[J]. Heilongjiang Textile, 2014, (23):58-58.

[9] S Q Fu, Y P Zhang. Discuss on the reliability design technologies of electeical communication equipment [J]. Electronic product reliability and environmental testing, 2014, (16):274-274.

[10] R.A. Bakkiyaraj, N. Kumarappan. Reliability design of composite generation and transmission system based on Latin Hyperc ube Sampling with GRNN state adequacy evaluation[C]. 2013 IET Chennai Fourth International Conference on Sustainable Energy and Intelligent Systems (SEISCON 2013):8 - 14 . 